\title{
Asymptotically charged BTZ Black Holes in Gravity's Rainbow
}

Received: / Accepted:

\begin{abstract}
Motivated by the wide applications of BTZ black holes and interesting results of gravity's rainbow, we consider three dimensional rainbow solutions and investigate their thermodynamic properties. In addition to investigate black holes thermodynamics related to AdS/CFT correspondence, one may regard gravity's rainbow to to encode quantum gravity effects into the black hole solutions. We take into account the various models of linear and nonlinear electrodynamics and study their effects on the gravity's rainbow spacetime. We also examine thermal stability and find that obtained three dimensional rainbow black holes are thermally stable.
\end{abstract}

\section{Introduction}

One of the weighty dream of physicists is creating a consistent quantum theory of gravity. Although various aspects of the quantum gravity is still very much alive, till now, all the attempts of obtaining a complete description of the quantum gravity have been unfeasible. Gravity's rainbow arises from deep attempts to fix the gaps between the theory of general relativity and quantum mechanics. One of the possible interesting consequence of gravity's rainbow is that the universe had no beginning, and therefore time is stretched back infinitely without encountering a big bang [1]. Also, thermodynamical properties of black p-branes was reported within the framework of gravity's rainbow [2]. Furthermore, as more recent application of gravity's rainbow, a recent investigation on the possibility of resolving the black hole information paradox was carried out in Ref. 3. In addition, the effects of the gravity's rainbow on the deflection of light, photon time delay, gravitational red-shift, and the weak equivalence principle have been studied in Ref. [4. Other consequences of the gravity's rainbow in the context of the gravitational collapse and black hole physics have been investigated in literature (see [5, 6, ,7,, 8 , and references therein).

On the other hand, some of theoreticians believe that the violation of Lorentz invariancy is an essential rule to construct quantum theory of gravity. The Lorentz invariance violation may be expressed in form of modified dispersion relations [9,10,11,12,13. In other words, according to the loop quantum gravity (LQG) results [10] and spacetime discreteness assumption [13] at the Planck scale, one may regard Lorentz invariance violation or modified dispersion relation by redefining the physical momentum and energy. In order to have a deep insight into the Lorentz invariancy, one may regard the close relation between gravity's rainbow and Horava-Lifshitz gravity 14. This relation comes from the fact that both these theories are based on the breaking of the usual energy-momentum dispersion relation in the UV limit in such a way that it reduces to the usual energy-momentum dispersion relation in

Present address: hendi@shirazu.ac.ir

\footnotetext{
${ }^{1}$ Physics Department and Biruni Observatory, College of Sciences, Shiraz University, Shiraz 71454, Iran

${ }^{2}$ Research Institute for Astrophysics and Astronomy of Maragha (RIAAM), P.O. Box 55134-441, Maragha, Iran
} 
the IR limit. The UV modification of the usual energy-momentum relation implies the breaking of the Lorentz symmetry. Due to the existence of an unstable perturbative string vacuum, the spontaneous breaking of the Lorentz symmetry can be occurred in string theory 9. In addition, the Lorentz symmetry breaking can be studied using black brane in the context of type IIB string theory [15. Taking into account the mentioned motivation of UV deformation of geometries that occur in string theory and the close relation between Horava-Lifshitz gravity and gravity's rainbow 14, recently rainbow deformation of geometries has been performed, in which leads to modification of the dispersion relation. One of the modified dispersion relation, which is predicted by new approach of quantum gravity [16, 17], takes the following general form

$$
f^{2}(\varepsilon) E^{2}-g^{2}(\varepsilon) p^{2}=m^{2},
$$

where dimensionless relative energy $\varepsilon=E / E_{p}$, in which $E$ is the energy of the probing particle and $E_{p}$ is the Planck energy. In addition, temporal and spatial rainbow functions $(f(\varepsilon)$ and $g(\varepsilon))$ satisfy the following conditions

$$
\lim _{\varepsilon \rightarrow 0} f(\varepsilon)=1, \quad \lim _{\varepsilon \rightarrow 0} g(\varepsilon)=1 .
$$

On the other hand, such modified dispersion relation can be originated from the so-called doubly special relativity 18. Doubly special relativity is a natural extension of special relativity, which enjoys the invariancy of the speed of light, to the case of assuming the invariancy of the maximum energy scale (the Planck energy) or equivalently minimum length scale [19]. Besides, the generalized uncertainty principle (GUP) which comes from various approaches to quantum gravity, is based on the existence of minimum measurable length scale $20,21,22,23,24,25,26$. Such minimum length scale has been applied to black hole thermodynamics $27,28,29,30,31$. From string theory point of view, it is not possible to probe spacetime below the string length scale and one can translate the minimum length scale into a maximum energy scale $32,33,34,35,36,37$. Including the curvature into the doubly special relativity, Magueijo and Smolin proposed the so-called doubly general relativity 38. In other words, doubly general relativity is a natural extension of doubly special relativity to the case of assuming curved spacetime. Regarding the doubly general relativity, one finds the geometry of spacetime may depend on the energy of the particle moving in it. In other words, spacetime is parameterized by the ratio $\varepsilon=E / E_{P}$ to obtain a parametric family of metrics, the so-called rainbow of metrics 38 . Hence, the modified metric in gravity's rainbow can be written as 38

$$
g(\varepsilon)=\eta^{a b} e_{a}(\varepsilon) \otimes e_{b}(\varepsilon),
$$

in which the energy dependence of the frame fields is

$$
e_{0}(\varepsilon)=\frac{1}{f(\varepsilon)} \hat{e}_{0}, \quad e_{i}(\varepsilon)=\frac{1}{g(\varepsilon)} \hat{e}_{i},
$$

where the hatted quantities refer to the energy independent frame fields. In Ref. 8] it was shown that one can explain the absence of black holes at LHC by using the gravity's rainbow. In addition, regarding gravity's rainbow, one may resolve the black hole information paradox without any need for black hole complementarity or Firewall [3. The functional forms of $f(\varepsilon)$ and $g(\varepsilon)$ are based on various phenomenological motivations [1,3,39,40,41,42,43,44,45]. Among different proposals in the literature, we point out three interesting models of modified dispersion relation.

One of the most studied models was proposed in Refs. [46,47]. This model, which we identify it as first model, is compatible with the results of LQG and non-commutative spacetime [47. In this model one should set the temporal rainbow's function to unity and spatial one is a square root function with the following explicit forms

$$
f(\varepsilon)=1, \quad g(\varepsilon)=\sqrt{1-\eta \varepsilon^{n}},
$$

Amelino-Camelia, et al. proposed another model in Ref. 12, which we will call second model. The hard spectra of gamma-ray bursters may be explained by the second model [12. In this model one sets spatial rainbow function to unity and temporal one has exponential form

$$
f(\varepsilon)=\frac{e^{\sigma \varepsilon}-1}{\sigma \varepsilon}, \quad g(\varepsilon)=1,
$$


Another model, which we will recognize it as third model, is related to constant speed of light and one may use it to solve the horizon problem [38. In this model both rainbow's functions are the same with the following fractional form

$$
f(\varepsilon)=g(\varepsilon)=\frac{1}{1-\lambda \varepsilon} .
$$

Regarding the three mentioned models, it is notable that the dimensionless parameters $\eta, n, \sigma$ and $\lambda$ are of the order of unity.

Among all black hole solutions one can think of, BTZ (Banados- Teitelboim-Zanelli) [48,49] black hole can be considered as the unique one. It is believed that $(2+1)$-dimensional BTZ black hole solutions have great achievements and provide a simplified model to find some conceptual issues in the context of black hole physics, quantum gravity, string and gauge theory, and also in the context of the AdS/CFT correspondence [50,51,52,53,54,55,56. In addition, critical behavior of the BTZ black hole with torsion has been considered in Ref. [57. Also, Gregory-Laflamme instability of BTZ black hole in the context of massive gravity has been reported by Moon and Myung 58. Thermodynamic equilibrium states of a typical static thin shell in a $(2+1)$-dimensional spacetime have been analyzed in 59] and thermodynamical properties of a BTZ black hole solution with an Horndeski source have been investigated in Ref. [60. For more subjects related to BTZ solutions, we refer the reader to Refs. $61,62,63,64,65,66,67,68,69,70,71,72$ and references therein.

Recently, the effective potential structure of the neutral BTZ black hole in gravity's rainbow was studied in Ref. 73. In this paper, we develop BTZ solutions to the case of existence of electrodynamics. We take into account the linear Maxwell field as well as some of the known nonlinear electrodynamics models in gravity's rainbow. We also investigate thermodynamical behavior of the solutions and discuss thermal stability of them.

\section{BTZ and asymptotically BTZ Gravity's Rainbow}

The Lagrangian of Einstein gravity with cosmological constant coupled to an electromagnetic field may be written as

$$
L_{\text {tot }}=R-2 \Lambda-\mathcal{L}(\mathcal{F}),
$$

where $R$ and $\Lambda$ are, respectively, the Ricci scalar and the cosmological constant, and electromagnetic Lagrangian, $\mathcal{L}(\mathcal{F})$, is a function of Maxwell invariant $\mathcal{F}=F_{a b} F^{a b}$, where the Faraday tensor is $F_{a b}=$ $\partial_{[a} A_{b]}$ and $A_{b}$ is the gauge potential. Taking into account the gauge-gravity Lagrangian (8) and applying the variational method, we obtain

$$
\begin{gathered}
G_{a b}+\Lambda g_{a b}=T_{a b}, \\
\nabla_{a}\left(\mathcal{L}_{\mathcal{F}} F^{a b}\right)=0,
\end{gathered}
$$

where $G_{a b}$ is the Einstein tensor, $\mathcal{L}_{\mathcal{F}}=\frac{d \mathcal{L}(\mathcal{F})}{d \mathcal{F}}$ and

$$
T_{a b}=\frac{1}{2} g_{\mu \nu} L(\mathcal{F})-2 F_{\mu \lambda} F_{\nu}^{\lambda} L_{\mathcal{F}},
$$

In this paper, in addition to the linear Maxwell electrodynamics (MED), we consider four classes of NED fields, namely BI nonlinear electrodynamics (BINED), Exponential form of nonlinear electrodynamics (ENED), Logarithmic form of nonlinear electrodynamics (LNED) and Power-Maxwell invariant form of electrodynamics (PNED) in which their Lagrangians are $74,75,76,77,78,79,80,81,82,83,84$, 85

$$
L(\mathcal{F})=\left\{\begin{array}{cc}
-\mathcal{F}, & M E D \\
4 \beta^{2}\left(1-\sqrt{1+\frac{\mathcal{F}}{2 \beta^{2}}}\right), & \text { BINED } \\
\beta^{2}\left(\exp \left(-\frac{\mathcal{F}}{\beta^{2}}\right)-1\right), & E N E D . \\
-8 \beta^{2} \ln \left(1+\frac{\mathcal{F}}{8 \beta^{2}}\right), & L N E D \\
(-\mathcal{F})^{\alpha}, & P N E D
\end{array}\right.
$$


In these equations, $\beta$ and $\alpha$ are called the nonlinearity parameters. It is notable that for $\beta \longrightarrow \infty$ and $\alpha \longrightarrow 1$, all the mentioned nonlinear models reduce to Maxwell Lagrangian.

Before we proceed, we would like to provide some reasonable motivations for considering these forms of NED.

Although Maxwell theory is capable for describing different phenomena in classical electrodynamics, it is not flawless and has some fundamental problems [86, 87. In order to solve these problems, we can generalize Maxwell theory to the case of NED [76,77,78,79,80,81, 82, 83, 84, 85, 86, 87, 88, Another strong motivation of considering NED, comes from developments in string/M-theory. It has been shown that the Born-Infeld [74 (BI)-type theories are specific in the context of NED models, which are naturally acquired in the low-energy limit of heterotic string theory [89,90,91,92. In addition, it is easy to show that the weak field limit of BI-type models is the same as calculation of the oneloop approximation of QED, which leads to quadratic Maxwell invariant in addition to the Maxwell Lagrangian [93].

Recently two BI-types of NED have been introduced, in which we can remove the divergency of the electric field of point-like charge near the origin. It was shown that BI-types of NED enjoy special and important properties. To name a few, one can indicate the absence of shock waves, birefringence 94, 95 and an electric - magnetic duality [96. It was shown that in a uniform electromagnetic field background for electrons, calculation of exact one-loop corrections leads to presence of logarithmic form of electromagnetic field strength [97. This correction term comes from the vacuum polarization effects. On the other hand, BI-type theories have been employed for explaining the equation of state of radiation for inflation 88. In addition, in the context of the holographic superconductors, it was shown that these NED models make significant effects on condensation, critical temperature of the superconductor and its energy gap [see [98, 99, 100, 101,102, for more details]. It was shown that the exponential form of NED has stronger effect on the condensation formation and conductivity comparing to logarithmic form of NED [101, 102].

Now let us present some motivations of considering PMI theory. The PMI theory is significantly richer than the Maxwell field, and in the special case $(s=1)$ it reduces to linear electrodynamics 82 , 83, 84, 85. One of the most important properties of the PMI model in $d$-dimensions occurs for $s=d / 4$ where the PMI theory becomes conformally invariant and so the energy-momentum tensor will be traceless (the same as Maxwell theory in four-dimensions [79,80,81]). In this case, one can obtain an inverse square law for the electric field of point-like charge in arbitrary dimensions. Furthermore, it was shown that there is an interesting relation between the black hole solutions of a class of pure $F(R)$ gravity and those of conformally invariant Maxwell source in Einstein gravity [103. In the context of AdS/CFT correspondence, the effects of PMI source on strongly coupled dual gauge theory have been investigated [104] (for more motivations of PMI theory, we refer the reader to [105]). Motivated by the recent results mentioned above, we consider the linear and nonlinear electrodynamics in three dimensional gravity's rainbow.

It was shown that adding the curvature to doubly special relativity (DSR) 18 leads to an extension, called doubly general relativity (DGR) 38. In DGR the spacetime geometry depends on the energy of the particle $(E)$. Thus, one may regard a spacetime which is parameterized by the energy ratio $\varepsilon$ to form a rainbow of metrics. Three dimensional static metric in the framework of gravity's rainbow is given by $[38$

$$
d \tau^{2}=-d s^{2}=\frac{\Psi(r)}{f^{2}(\varepsilon)} d t^{2}-\frac{1}{g^{2}(\varepsilon)}\left(\frac{d r^{2}}{\Psi(r)}+r^{2} d \phi^{2}\right) .
$$

In this paper, we are looking for the black hole solutions with a radial electric field. Thus the nonzero components of the gauge potential is temporal component. Regarding the mentioned models of Eq. (12) and using the gauge potential $A_{\mu}=h(r) \delta_{\mu}^{0}$, we find that the electromagnetic field equation 
(10) leads to the following differential equations

$$
\begin{array}{ll}
r h^{\prime \prime}+h^{\prime}=0, & M E D \\
r h^{\prime \prime}+h^{\prime}\left[1-\left(\frac{f(\varepsilon) g(\varepsilon) h^{\prime}}{\beta}\right)^{2}\right]=0, & B I N E D \\
r\left[1+\left(\frac{2 f(\varepsilon) g(\varepsilon) h^{\prime}}{\beta}\right)^{2}\right] h^{\prime \prime}+h^{\prime}=0, & \text { ENED } \\
r h^{\prime \prime}\left[1+\left(\frac{f(\varepsilon) g(\varepsilon) h^{\prime}}{2 \beta}\right)^{2}\right]+ & \\
{\left[1-\left(\frac{f(\varepsilon) g(\varepsilon) h^{\prime}}{2 \beta}\right)^{2}\right] h^{\prime}=0,} & \text { LNED } \\
r(2 \alpha-1) h^{\prime \prime}+h^{\prime}=0, \quad(\alpha \neq 1) & P N E D
\end{array}
$$

where prime and double primes denote first and second derivative with respect to $r$, respectively. The solutions of the mentioned differential equations are

$$
h(r)=\left\{\begin{array}{cc}
q \ln \left(\frac{r}{l}\right), & M E D \\
q \ln \left[\frac{r}{2 l}(1+\Gamma)\right], & B I N E D \\
q\left[\exp \left(-\frac{L_{W}}{2}\right)+\frac{E_{i}\left(1, \frac{L_{W}}{2}\right)}{2}+,\right. & E N E D \\
\left.\ln \left(\frac{\sqrt{2} f(\varepsilon) g(\varepsilon) q}{\beta l}\right)+\frac{\gamma}{2}-1\right] & \\
\frac{\beta^{2} r^{2}(\Gamma-1)}{q f(\varepsilon)^{2} g(\varepsilon)^{2}}-\frac{q}{2}+q \ln \left[\frac{r(1+\Gamma)}{2 l}\right], & L N E D \\
\frac{(2 s-1) q}{2(s-1) r^{\frac{2(1-s)}{2 s-1}},} \quad(s \neq 1) & P N E D
\end{array}\right.
$$

where $\Gamma=\sqrt{1+\frac{f^{2}(\varepsilon) g^{2}(\varepsilon) q^{2}}{r^{2} \beta^{2}}}, q$ is an integration constant which is related to the electric charge of the black hole. In addition, $L_{W}=\operatorname{Lambert} W\left(\frac{4 f^{2}(\varepsilon) g^{2}(\varepsilon) q^{2}}{\beta^{2} r^{2}}\right)$ which satisfies LambertW $(x) \exp [\operatorname{Lambert} W(x)]=$ $x, \gamma=\gamma(0) \simeq 0.57722$ and the special function $E i(1, x)=\int_{1}^{\infty} \frac{e^{-x z}}{z} d z$ (for more details, see [106]).

According to the gauge potential ansatz with Eq. (15), one finds that the non-vanishing components of the electromagnetic field tensor can be written as

$$
F_{t r}=-F_{r t}=\left\{\begin{array}{cc}
\frac{q}{r}, & M E D \\
\frac{q}{r \Gamma}, & B I N E D \\
\frac{q}{r} \exp \left(-\frac{L_{W}}{2}\right), & E N E D \\
\frac{2 r \beta^{2}}{q f^{2}(\varepsilon) g^{2}(\varepsilon)}(\Gamma-1), & L N E D \\
\frac{q}{r^{\frac{1}{2 \alpha-1}}}, & P N E D
\end{array}\right.
$$

In order to have a sensible asymptotic structure for the electromagnetic field, $F_{t r}$ should vanish for large values of $r$. This condition is satisfied for MED, BINED, ENED and LNED branches. For PNED case, vanishing $F_{t r}$ for $r \rightarrow \infty$ leads to $\alpha>1 / 2$. Thus in this paper, we restrict ourselves to $\alpha>1 / 2$. 
Regarding the gravitational field equation for the mentioned models of NED, we find that the following metric function satisfies all components of the field equation (9), simultaneously

$$
\Psi(r)=-\frac{\Lambda r^{2}}{g(E)^{2}}-m+\Theta(r),
$$

where

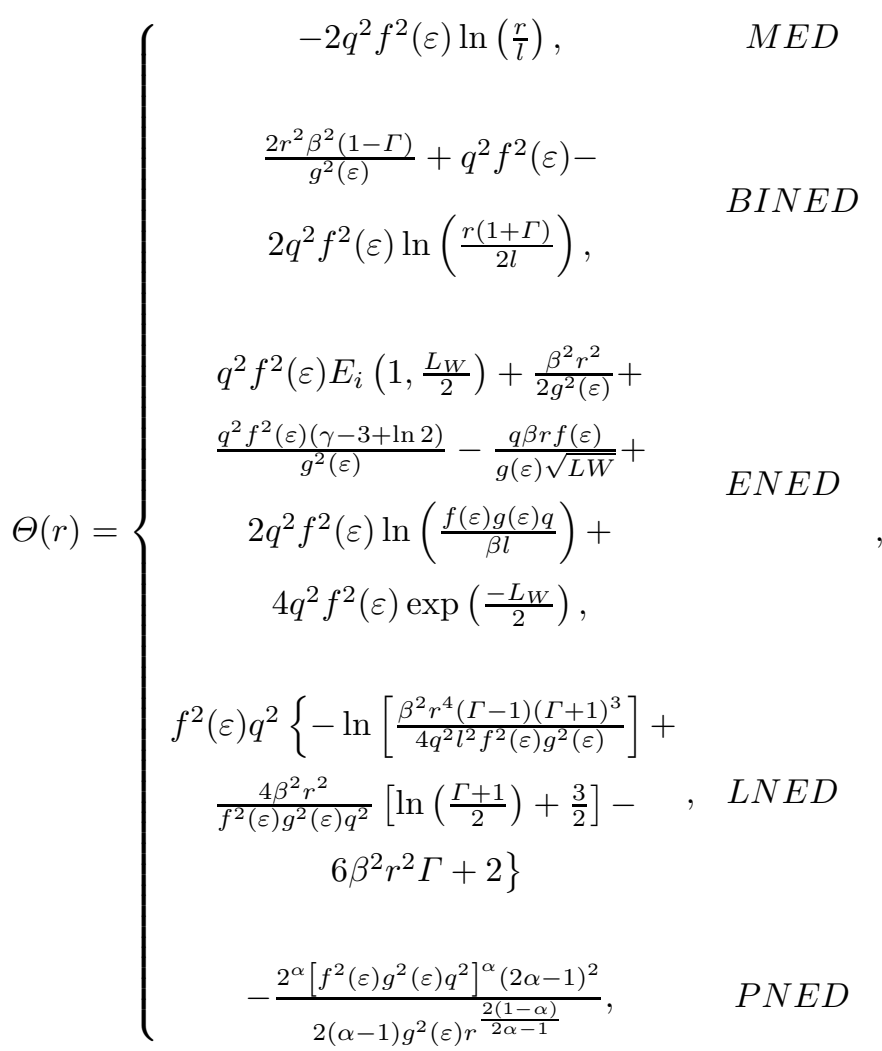

and $m$ is an integration constant which is related to the total mass $(M)$

$$
M=\frac{m}{8 f(\varepsilon)}
$$

It is notable that the ArnowittDeserMisner (ADM) mass of black hole can be obtained by using the behavior of the metric at large $r$. Straightforward calculations show that ADM mass of the black hole solutions is the same as Eq. (19).

Using series expansion of metric functions for large values of $r$, we find that $\Lambda$-term will be dominated and therefore obtained solutions are asymptotically AdS with an effective cosmological constant, $\Lambda_{e f f}=\frac{\Lambda}{g(E)^{2}}$. In addition, calculations show that the Ricci and the Kretschmann scalars for the metric (13) are

$$
\begin{gathered}
R=-g^{2}(\varepsilon) \Psi^{\prime \prime}-\frac{2 g^{2}(\varepsilon) \Psi^{\prime}}{r} \\
R_{\mu \nu \rho \sigma} R^{\mu \nu \rho \sigma}=g^{4}(\varepsilon) \Psi^{\prime \prime 2}+2\left(\frac{g^{2}(\varepsilon) \Psi^{\prime}}{r}\right)^{2} .
\end{gathered}
$$

Inserting Eq. (17) into the Eqs. (20) and (21), we find that there is an essential singularity located at $r=0$. Depending on the values of free parameters, one confirms that the mentioned singularity can be covered with an event horizon [76. Applying the surface gravity interpretation on the event horizon, one finds the Hawking temperature as

$$
T=-\frac{\Lambda r_{+}}{2 \pi f(\varepsilon) g(\varepsilon)}+\Upsilon_{+}
$$


where

$$
\Upsilon_{+}=\left\{\begin{array}{cc}
\frac{-f(\varepsilon) g(\varepsilon) q^{2}}{2 \pi r_{+}}, & M E D \\
\frac{r \beta^{2}\left(1-\Gamma_{+}\right)}{\pi f(\varepsilon) g(\varepsilon)}, & B I N E D \\
\frac{q \beta\left(1-L_{W+}\right)}{2 \pi \sqrt{L_{W+}}-\frac{\beta^{2} r_{+}}{4 \pi f(\varepsilon) g(\varepsilon)},} & E N E D \\
\frac{2 \beta^{2} r_{+}\left[1+\ln \left(\frac{1+\Gamma_{+}}{2}\right)-\Gamma_{+}\right]}{\pi f(\varepsilon) g(\varepsilon)}, & L N E D \\
\frac{-\left[2 f^{2}(\varepsilon) g^{2}(\varepsilon) q^{2}\right]^{\alpha}(2 \alpha-1)}{4 \pi f(\varepsilon) g(\varepsilon) r_{+}^{\frac{1}{2 \alpha-1}}}, & P N E D
\end{array},\right.
$$

and $\Gamma_{+}=\sqrt{1+\frac{f^{2}(\varepsilon) g^{2}(\varepsilon) q^{2}}{r_{+}^{2} \beta^{2}}}$ and $L_{W_{+}}=\operatorname{Lambert} W\left(\frac{4 f^{2}(\varepsilon) g^{2}(\varepsilon) q^{2}}{\beta^{2} r_{+}^{2}}\right)$.

It is worthwhile to mention that in the absence of rainbow's effects $(f(\varepsilon)=g(\varepsilon)=1)$, there is a critical value for the horizon radius $\left(r_{+c}\right)$, in which the temperature is positive for $r_{+}>r_{+c}$. The value of $r_{+c}$ depends on the choices of other parameters. In order to have a consistent limitation, we restrict $f(\varepsilon)$ and $g(\varepsilon)$ to smooth positive functions. It is evident that due to the presence of rainbow's functions, the behavior of temperature and the values of $r_{+c}$ change, drastically.

\section{First law of thermodynamics and thermal stability}

In order to check the First law of thermodynamics, we should calculate entropy, electric potential and charge. Since we are working in Einstein gravity, it is allowed to use the area law to obtain the entropy [107, 108, 109]. The entropy of black holes is equal to one-quarter of the horizon area

$$
S=\frac{\pi r_{+}}{2 g(\varepsilon)}
$$

Besides, we can obtain the electric charge by using the Gauss law. By calculation of the flux of the electric field at infinity, one can find the electric charge as

$$
Q=\left\{\begin{array}{cc} 
& M E D \\
\frac{q f(\varepsilon)}{2}, & B I N E D \\
& E N E D \\
& L N E D \\
\frac{2^{\alpha-2} \alpha}{g(\varepsilon)}[q f(\varepsilon) g(\varepsilon)]^{2 \alpha-1}, & P N E D
\end{array}\right.
$$

where for $\alpha=1$, the electric charge of PNED branch is compatible with other branches. The electric potential $\Phi$, measured at the reference with respect to the horizon, is defined by

$$
\Phi=\left.A_{\mu} \chi^{\mu}\right|_{\text {reference }}-\left.A_{\mu} \chi^{\mu}\right|_{r=r_{+}},
$$


where $\Phi$ vanishes at the reference and $\chi^{\mu}$ is the null generator of the horizon, yielding

$$
\Phi=\left\{\begin{array}{cc}
-q \ln \left(\frac{r_{+}}{l}\right), & \text { MED } \\
-q \ln \left[\frac{r_{+}}{2 l}\left(1+\Gamma_{+}\right)\right], & \text {BINED } \\
q\left[1-\exp \left(-\frac{L_{W_{+}}}{2}\right)-\frac{\gamma}{2}-\right. & \\
\left.\ln \left(\frac{\sqrt{2} f(\varepsilon) g(\varepsilon) q}{\beta l}\right)-\frac{E_{i}\left(1, \frac{L_{W_{+}}}{2}\right)}{2}\right], & \text { ENED } \\
\frac{q}{2}+\frac{\beta^{2} r_{+}^{2}\left(1-\Gamma_{+}\right)}{q f^{2}(\varepsilon) g^{2}(\varepsilon)}-q \ln \left[\frac{\left(1+\Gamma_{+}\right) r_{+}}{2 l}\right], & \text { LNED } \\
\frac{-(2 \alpha-1) q}{2(\alpha-1) r_{+}^{2(1-\alpha)}},(\alpha \neq 1) & P N E D
\end{array}\right.
$$

After calculating all of the conserved and thermodynamic quantities of the solutions, we are in a position to examine the first law of thermodynamics. To do this, we obtain the total mass $M$ as a function of the extensive quantities $Q$ and $S$. Using the expression for the entropy, the electric charge and the mass given in Eqs. (17), (19), (24) and (25), and the fact that $f\left(r=r_{+}\right)=0$, one can obtain a Smarr-type formula as

$$
M(S, Q)=-\frac{\Lambda S^{2}}{2 \pi^{2} f(\varepsilon)}-\frac{\Sigma}{f(\varepsilon)},
$$

where

$$
\Sigma=\left\{\begin{array}{cc}
Q^{2} \ln \left(\frac{2 g(\varepsilon) S}{\pi l}\right), & M E D \\
Q^{2} \ln \left(\frac{g(\varepsilon)\left(1+\Gamma^{\prime}\right) S}{\pi l}\right)-2\left(\frac{\beta\left(1-\Gamma^{\prime}\right) S}{2 \pi}\right)^{2}, & B I N E D \\
\frac{Q^{2}}{2}\left[E i\left(1, \frac{L_{W}^{\prime}}{2}\right)+\gamma-3\right]+ & E N E D \\
\frac{Q^{2}}{2} \ln \left(\frac{8 Q^{2} g^{2}(\varepsilon)}{\beta^{2} l^{2}}\right)+\frac{\beta^{2} S^{2}}{4 \pi^{2}}- & \\
\frac{\beta Q S\left(1-2 L_{W}^{\prime}\right)}{2 \pi \sqrt{L_{W}^{\prime}}}, & \\
\frac{Q^{2}}{8}\left[\frac{\left(3-\Gamma^{\prime 2}\right) \ln \left(\frac{1+\Gamma^{\prime}}{2}\right)}{\left(1-\Gamma^{\prime 2}\right)}+\frac{\left(2-\Gamma^{\prime}\right)}{\left(1+\Gamma^{\prime}\right)}+\right. & \\
\left.\ln \left(\frac{2 g(\varepsilon) S}{\pi l}\right)\right], & \\
\frac{(2 \alpha-1)^{2}\left(\frac{2^{\alpha} Q}{\alpha}\right)}{4(\alpha-1)\left(\frac{S}{\pi}\right)^{\frac{2-2 \alpha}{2 \alpha-1}}}, & P N E D
\end{array}\right.
$$

and $\Gamma^{\prime}=\sqrt{1+\frac{\pi^{2} Q^{2}}{\beta^{2} S^{2}}}$ and $L_{W}^{\prime}=\operatorname{Lambert} W\left(\frac{4 \pi^{2} Q^{2}}{\beta^{2} S^{2}}\right)$. Taking into account Eq. (28), we can calculate the temperature and electric potential as intensive parameters

$$
T=\left(\frac{\partial M}{\partial S}\right)_{Q}, \quad \Phi=\left(\frac{\partial M}{\partial Q}\right)_{S},
$$

which are the same as those calculated in Eqs. (22) and (26) (for all branches). Therefore we conclude that obtained conserved and thermodynamic quantities satisfy the first law of thermodynamics

$$
d M=T d S+\Phi d Q .
$$


In what follows, we investigate thermal stability. In principle, thermal stability of a system with respect to small variations of the thermodynamic coordinates is usually performed by investigating the behavior of the entropy $S(M, Q)$ around the equilibrium.

The local stability criteria in any ensemble requires that $S(M, Q)$ be a smooth convex function of the extensive variables or its Legendre transformation should be a smooth concave function of the intensive thermodynamic coordinates. Thermal stability can also be studied by the behavior of the total mass $M(S, Q)$ which should be a smooth convex function of its extensive variables. Therefore, the local stability can in principle be carried out by obtaining the determinant of the Hessian matrix of $M(S, Q)$ versus its extensive coordinates $X_{i}, \mathbf{H}_{X_{i} X_{j}}^{M}=\left[\partial^{2} M / \partial X_{i} \partial X_{j}\right]$ (see Refs. [110,111,112] for more details). In our case the mass $M$ is a function of the entropy $S$ and the charge $Q$. The number of thermodynamic variables depends on the ensemble that is used. In what follows, we calculate the heat capacity of (nonlinearly) charged black hole solutions to investigate thermal stability through canonical ensemble. We should regard the electric charge as a fixed parameter and calculate $C_{Q}=T\left(\frac{\partial S}{\partial T}\right)_{Q}$. The positivity of the heat capacity guarantees the local thermal stability. Since we investigate the physical black hole solutions (positive temperature), it is sufficient to examine the positivity of $\left(\frac{\partial S}{\partial T}\right)_{Q}=\left(\frac{\partial^{2} M}{\partial S^{2}}\right)_{Q}^{-1}$ for thermal stability. Calculations show that

$$
\left(\frac{\partial^{2} M}{\partial S^{2}}\right)_{Q}=\frac{-\Lambda}{\pi^{2} f(\varepsilon)}+\left\{\begin{array}{cc}
\frac{q^{2} f(\varepsilon) g^{2}(\varepsilon)}{\pi^{2} r_{+}^{2}}, & M E D \\
\frac{2 \beta^{2}\left(\Gamma_{+}-1\right)}{\pi^{2} f(\varepsilon) \Gamma_{+}}, & B I N E D \\
\frac{\beta^{2}\left(e^{\frac{L_{W+}}{2}}-1\right)}{2 \pi^{2} f(\varepsilon)}, & E N E D \\
\frac{4 \beta^{2} \ln \left(\frac{1+\Gamma_{+}}{2}\right)}{\pi^{2} f(\varepsilon)}, & L N E D \\
\frac{2^{\alpha}[q f(\varepsilon) g(\varepsilon)]^{2 \alpha}}{2 \pi^{2} f(\varepsilon) r_{+}^{\frac{2 \alpha}{2 \alpha-1}}}, & P N E D
\end{array}\right.
$$

It is clear to find that $\Gamma_{+}$and $e^{\frac{L_{W+}}{2}}$ are greater than one and therefore $\left(\frac{\partial^{2} M}{\partial S^{2}}\right)_{Q}$ is positive definite for obtained AdS solutions. Regarding positive values for $f(\varepsilon)$ and $g(\varepsilon)$ and taking into account a positive temperature for the physical black hole solutions ( for $r_{+}>r_{+c}$ ), we can conclude that the mentioned black holes enjoy thermal stability. In comparison with the asymptotically AdS black holes of Einstein gravity, which have a small unstable phase, the stability phase structure of the Einstein(nonlinear) Maxwell black holes in gravity's rainbow shows that the electric charge help us to obtain stable solutions and rainbow functions do not make anomaly.

\section{Note on the integration constants}

Regarding positive real rainbow functions with the functional forms of $\Gamma$ and $L_{W}$ (after Eq. (15)), one finds that there is no restriction on $q$ and $m$. Besides, one can follow the method of Refs. [113, 114, 115. with a specific model of rainbow function to obtain possible mass/charge limitations. Since in this paper we have discussed general form of rainbow functions, we release the mentioned method.

Taking into account Eqs. (15) and (17), one can find that $q$ and $m$ are integration constant. Since the variable of integrations is $r$ coordinate, we can replace $q$ and $m$ with modified constants such as $q f^{a}(\varepsilon) g^{b}(\varepsilon)$ and $m f^{c}(\varepsilon) g^{d}(\varepsilon)$, respectively ( $a, b, c$ and $d$ are arbitrary real valued numbers). It is worthwhile to mention that although in new situation, all conserved and thermodynamic quantities will be modified, the results for $\varepsilon \rightarrow 0(f(\varepsilon)=g(\varepsilon)=1)$ do not change. Another comment which should be discussed is the first law and thermal stability. Regarding new situation (applying $q \rightarrow q f^{a}(\varepsilon) g^{b}(\varepsilon)$ and $m \rightarrow m f^{c}(\varepsilon) g^{d}(\varepsilon)$ in previous sections), it is easy to show that the first law is satisfied. In addition, the criteria of thermal stability do not change, globally. These behaviors are expected, since we applied a 
global transformation on the constants. Considering new situation, one can obtain four free parameters $a, b, c$ and $d$, and their manipulations with special choices may lead to interesting results.

\section{Closing Remarks}

In this paper, we took into account the gravity's rainbow in three dimensions in the presence of linear and nonlinear models of electrodynamics. The motivation of considering gravity's rainbow comes from the quantum gravity view point, while the reason of considering nonlinear electrodynamics is arisen from LQG as well as low effective limit of heterotic sting theory. We found that although the rainbow's functions affected the electromagnetic field behavior of nonlinear BI-type models, Maxwell and PNED models of electromagnetic field did not depend on the rainbow's functions. We obtained metric functions of each models and found that these solutions can be interpreted as black holes.

Then, we calculated conserved and thermodynamic quantities and found that these solutions may depend on the choice of rainbow's functions. We also considered finite mass as a function of extensive parameters and checked the first law of thermodynamics. In addition, we analyzed thermal stability by using of the heat capacity. We found that obtained physical solutions $(T>0)$ enjoyed thermal stability in the canonical ensemble.

It is worthwhile to generalize these three dimensional solutions to four and higher dimensional cases with various horizon topologies. It is also interesting to investigate the effects of rainbow's functions on dilaton gravity as well as higher curvature theory. These cases will be addressed elsewhere.

\section{6 acknowledgements}

We are indebted to Mir Faizal for useful discussions and S. Panahiyan for reading the manuscript. We also wish to thank Shiraz University Research Council. This work has been supported financially by Research Institute for Astronomy and Astrophysics of Maragha.

\section{References}

1. A. Awad, A. F. Ali and B. Majumder, JCAP 1310, 052 (2013).

2. A. Ashour, M. Faizal, A. F. Ali and F. Hammad, arXiv:1602.04926.

3. A. F. Ali, M. Faizal and B. Majumder, Europhys. Lett. 109, 20001 (2015).

4. A. F. Ali and M. M. Khalil, Europhys. Lett. 110, 20009 (2015).

5. A. F. Ali, M. Faizal and M. M. Khalil, JHEP 1412, 159 (2014).

6. A. F. Ali, M. Faizal and M. M. Khalil, Nucl. Phys. B 894, 341 (2015).

7. A. F. Ali, M. Faizal, B. Majumder and R. Mistry, Int. J. Geom. Meth. Mod. Phys. 12, 1550085 (2015).

8. A. F. Ali, M. Faizal and M. M. Khalil, Phys. Lett. B 743, 295 (2015).

9. V. A. Kostelecky and S. Samuel, Phys. Rev. D 39, 683 (1989).

10. R. Gambini and J. Pullin, Phys. Rev. D 59, 124021 (1999).

11. S. M. Carroll, J. A. Harvey, V. A. Kostelecky, C. D. Lane and T. Okamoto, Phys. Rev. Lett. 87, 141601 (2001).

12. G. Amelino-Camelia, J. R. Ellis, N. E. Mavromatos, D. V. Nanopoulos and S. Sarkar, Nature 393, 763 (1998).

13. G. tHooft, Class. Quantum Gravit. 13, 1023 (1996).

14. R. Garattini and E. N. Saridakis, Eur. Phys. J. C 75, 343 (2015).

15. S. Mukohyama, JHEP 0705, 048 (2007).

16. P. Horava, Phys. Rev. D 79, 084008 (2009).

17. P. Horava, Phys. Rev. Lett. 102, 161301 (2009).

18. G. Amelino-Camelia, Int. J. Mod. Phys. D 11, 35 (2002).

19. J. Magueijo and L. Smolin, Phys. Rev. Lett. 88, 190403 (2002).

20. M. Faizal, Int. J. Geom. Meth. Mod. Phys. 12, 1550022 (2015).

21. M. Faizal, A. F. Ali and A. Nassar, J. Mod. Phys. A 30, 1550183 (2015).

22. A. F. Ali, M. Faizal and M. M. Khalil, JCAP 1509, 025 (2015).

23. M. Faizal and B. Majumder, Ann. Phys. 357, 49 (2015).

24. S. Pramanik, M. Moussa, M. Faizal and A. F. Ali, Ann. Phys. 362, 24 (2015).

25. A. Mohammadi, A. F. Ali, T. Golanbari, A. Aghamohammadi, Kh. Saaidi and M. Faizal, [1505.04392].

26. M. Faizal and S. I. Kruglov, Int. J. Mod. Phys. D 25, 1650013 (2016).

27. A. N. Tawfik and E. A. El Dahab, Int. J. Mod. Phys. A 30, 1550030 (2015).

28. S. Gangopadhyay, A. Dutta and M. Faizal, Europhys. Lett. 112, 20006 (2015).

29. P. Bargueno and E. C. Vagenas, Phys. Lett. B 742, 15 (2015). 
30. B. Carr, J. Mureika and P. Nicolini, JHEP 1507, 05 (2015).

31. F. Hammad, Phys. Rev. D 92, 044004 (2015).

32. M. Faizal and B. P. Mandal, Gravit. Cosmol. 21, 270 (2015).

33. M. Faizal, M. M. Khalil, S. Das, Eur. Phys. J. C 76, 30 (2016).

34. D. Amati, M. Ciafaloni and G. Veneziano, Phys. Lett. B 216, 41 (1989).

35. L. J. Garay, Int. J. Mod. Phys. A 10, 145 (1995).

36. A. F. Ali, S. Das and E. C. Vagenas, Phys. Lett. B 678, 497 (2009).

37. A. F. Ali, S. Das and E. C. Vagenas, Phys. Rev. D 84, 044013 (2011).

38. J. Magueijo and L. Smolin, Class. Quantum Gravit. 21, 1725 (2004).

39. R. Garattini and G. Mandanici, Phys. Rev. D 85, 023507 (2012).

40. C. Leiva, J. Saavedra and J. Villanueva, Mod. Phys. Lett. A 24, 1443 (2009).

41. H. Li, Y. Ling and X. Han, Class. Quantum Gravit. 26, 065004 (2009).

42. A. F. Ali, Phys. Rev. D 89, 104040 (2014).

43. J. D. Barrow and J. Magueijo, Phys. Rev. D 88, 103525 (2013).

44. C. Z. Liu and J. Y. Zhu, Gen. Relativ. Gravit. 40, 1899 (2008).

45. S. H. Hendi and M. Faizal, Phys. Rev. D 92, 044027 (2015)

46. G. Amelino-Camelia, J. R. Ellis, N. Mavromatos and D. V. Nanopoulos, Int. J. Mod. Phys. A 12, 607 (1997).

47. G. Amelino-Camelia, Living Rev. Relativ. 16, 5 (2013).

48. M. Banados, C. Teitelboim and J. Zanelli, Phys. Rev. Lett. 69, 1849 (1992)

49. M. Banados, M. Henneaux, C. Teitelboim and J. Zanelli, Phys. Rev. D 48, 1506 (1993).

50. S. Carlip, Class. Quantum Gravit. 12, 2853 (1995).

51. E. Witten, Adv. Theor. Math. Phys. 2, 505 (1998)

52. A. Ashtekar, J. Wisniewski and O. Dreyer, Adv. Theor. Math. Phys. 6, 507 (2002).

53. S. Carlip, Class. Quantum Gravit. 22, R85 (2005).

54. T. Sarkar, G. Sengupta and B. Nath Tiwari, JHEP 0611, 015 (2006).

55. M. R. Setare, Eur. Phys. J. C 49, 865 (2007).

56. E. Witten, arXiv:0706.3359.

57. M. S. Ma and R. Zhao, Phys. Rev. D 89, 044005 (2014).

58. T. Moon and Y. S. Myung, Phys. Rev. D 88, 124014 (2013).

59. J. P. S. Lemos and G. M. Quinta, Phys. Rev. D 89, 084051 (2014).

60. M. Bravo-Gaete and M. Hassaine, Phys. Rev. D 90, 024008 (2014).

61. T. G. Mertens, H. Verschelde and V. I. Zakharov, JHEP 1406, 156 (2014).

62. F. W. Shu, K. Lin, A. Wang and Q. Wu, JHEP 1404, 056 (2014).

63. D. V. Singh, Int. J. Mod. Phys. D 24, 1550001 (2015).

64. Y. Herrera, V. Hurovich, O. Santillan and C. Simeone, Phys. Rev. D 92, 085042 (2015).

65. J. P. S. Lemos, F. J. Lopes, M. Minamitsuji and Jorge V. Rocha, Phys. Rev. D 92, 064012 (2015).

66. S. H. Hendi, Eur. Phys. J. C 71, 1551 (2011).

67. S. H. Hendi, Prog. Theor. Phys. 127, 907 (2012).

68. S. H. Hendi, Int. J. Theor. Phys. 53, 4170 (2014).

69. S. H. Hendi, B. Eslam Panah and R. Saffari, Int. J. Mod. Phys. D 23, 1450088 (2014).

70. A. Sheykhi, S. H. Hendi, S. Salarpour, Phys. Scrip. 89, 105003 (2014).

71. S. H. Hendi, S. Panahiyan and B. Eslam Panah, Adv. High Energy Phys. 2015, 743086 (2015).

72. S. H. Hendi, S. Panahiyan and R. Mamasani, Gen. Relativ. Gravit. 47, 91 (2015).

73. C. Leiva and I. Espinoza, arXiv:1006.2406.

74. M. Born and L. Infeld, Proc. Roy. Soc. Lond. A 144, 425 (1934).

75. H. H. Soleng, Phys. Rev. D 52, 6178 (1995).

76. S. H. Hendi, JHEP 1203, 065 (2012).

77. S. H. Hendi, Ann. Phys. 333, 282 (2013).

78. S. H. Hendi, Ann. Phys. 346, 42 (2014).

79. M. Hassaine and C. Martinez, Phys. Rev. D 75, 027502 (2007).

80. S. H. Hendi and H. R. Rastegar-Sedehi, Gen. Relativ. Gravit. 41, 1355 (2009).

81. S. H. Hendi, Phys. Lett. B 677, 123 (2009).

82. M. Hassaine and C. Martinez, Class. Quantum Gravit. 25, 195023 (2008).

83. S. H. Hendi, Prog. Theor. Phys. 124, 493 (2010).

84. S. H. Hendi, Eur. Phys. J. C 69, 281 (2010).

85. S. H. Hendi, Phys. Rev. D 82, 064040 (2010).

86. D. H. Delphenich, arXiv:hep-th/0309108.

87. D. H. Delphenich, arXiv:hep-th/0610088

88. B. L. Altshuler, Class. Quantum Gravit. 7, 189 (1990)

89. E. S. Fradkin and A. A. Tseytlin, Phys. Lett. B 163, 123 (1985).

90. R. R. Matsaev, M. A. Rahmanov and A. A. Tseytlin, Phys. Lett. B 193, 207 (1987).

91. Y. Kats, L. Motl and M. Padi, JHEP 0712, 068 (2007).

92. R. G. Cai, Z. Y. Nie and Y. W. Sun, Phys. Rev. D 78, 126007 (2008).

93. A. Ritz and R. Delbourgo, Int. J. Mod. Phys. A 11, 253 (1996).

94. G. Boillat, J. Math. Phys. 11, 941 (1970).

95. G. Boillat, J. Math. Phys. 11, 1482 (1970).

96. G. W. Gibbons and D. A. Rasheed, Nucl. Phys. B 454, 185 (1995).

97. W. Heisenberg and H. Euler, Z. Phys. 98, 714 (1936).

98. J. Jing and S. Chen, Phys. Lett. B 686, 68 (2010). 
99. R. Gregory, S. Kanno and J. Soda, JHEP 0910, 010 (2009).

100. Q. Y. Pan, B. Wang, E. Papantonopoulos, J. Oliveira and A. Pavan, Phys. Rev. D 81, 106007 (2010).

101. S. Dey and A. Lala, Ann. Phys. 354, 165 (2015).

102. Z. Zhao, Q. Pan, S. Chen and J. Jing, Nucl. Phys. B 871, 98 (2013).

103. S. H. Hendi, Phys. Lett. B 690, 220 (2010).

104. J. Jing, Q. Pan and S. Chen, JHEP 1111, 045 (2011).

105. S. H. Hendi and M. H. Vahidinia, Phys. Rev. D 88, 084045 (2013).

106. R. M. Corless, G. H. Gonnet, D. E. G. Hare, D. J. Jeffrey and D. E. Knuth, Adv. Comput. Math. 5, 329 (1996).

107. J. D. Bekenstein, Lett. Nuovo Cim. 4, 737 (1972).

108. J. D. Bekenstein, Phys. Rev. D 7, 2333 (1973)

109. S. W. Hawking and C. J. Hunter, Phys. Rev. D 59, 044025 (1999).

110. M. Cvetic and S. S. Gubser, JHEP 9904, 024 (1999).

111. M. M. Caldarelli, G. Cognola and D. Klemm, Class. Quantum Gravit. 17, 399 (2000).

112. S. S. Gubser and I. Mitra, JHEP 0108, 018 (2001).

113. Y. Ling, X. Li and H. Zhang, Mod. Phys. Lett. A 22, 2749 (2007).

114. Y. Gim and W. Kim, JCAP 1410, 003 (2014).

115. B. Mu, P. Wang and H. Yang, JCAP 1511, 045 (2015). 